\title{
IDENTITIES IN IMPLICATIVE SEMILATTICES
}

\author{
ANGELO MARGARIS ${ }^{1}$
}

\begin{abstract}
An effective procedure is given for deciding whether or not an equation in the theory of implicative semilattices is an identity.
\end{abstract}

An implicative semilattice (Curry [1]) is a nonempty set $S$ together with a binary relation $\leqq$ in $S$, and two binary operations $\wedge$ and $\rightarrow$ in $S$, satisfying, for all $a, b, c$ in $S$ :

(1) $a \leqq a$.

(2) If $a \leqq b$ and $b \leqq c$, then $a \leqq c$.

(3) $a \wedge b \leqq a$ and $a \wedge b \leqq b$.

(4) If $c \leqq a$ and $c \leqq b$, then $c \leqq a \wedge b$.

(5) If $a \wedge b \leqq c$, then $a \leqq b \rightarrow c$.

(6) $a \wedge(a \rightarrow b) \leqq b$.

(7) If $a \leqq b$ and $b \leqq a$, then $a=b$.

$\leqq$ is a partial order by (1), (2) and (7); $a \wedge b$ is the greatest lower bound of $\{a, b\}$ by (3) and (4).

Let $a, b, c, d, e$ be elements of an implicative semilattice. The following properties are easily derived.

(8) $(a \rightarrow d) \wedge(a \rightarrow e)=a \rightarrow(d \wedge e)$.

(9) $(a \wedge d) \rightarrow e=a \rightarrow(d \rightarrow e)$.

(10) If $a \rightarrow c \leqq a$, then $a \rightarrow c \leqq c$.

A term is a meaningful expression built up from variables, $\wedge$ and $\rightarrow$ and parentheses. The rank of a term is the number of. occurrences of $\wedge$ and $\rightarrow$ in the term. If $a$ and $b$ are terms, then $a=b$ is an equation. An equation is an identity if and only if it holds in every implicative semilattice, or equivalently, if it follows from (1) through (7). The purpose of this paper is to give an effective procedure for deciding, for any terms $a$ and $b$, whether or not the equation $a=b$ is an identity.

It is easy to see that the problem of whether or not $a=b$ is an identity reduces to the problem of whether or not $a \leqq b$ and $b \leqq a$ are derivable from (1) through (6).

Received by the editors September 1, 1972 and, in revised form, February 2, 1973. AMS (MOS) subject classifications (1970). Primary 06A20, $02 \mathrm{E} 10$.

Key words and phrases. Decision procedure, identity, implicative semilattice.

1 Partially supported by an NSF COSIP grant. 
The decision procedure makes use of a relation $\subset$, which is not set inclusion, but an extension of $\leqq$. We first set the notation. $a, b, c, d, e$ are terms. $v$ is a variable. $R, S, T, U$ are finite (perhaps empty) sets of terms. $(S, T)$ is the union of $S$ and $T$. $(S, a, b)$ is the union of $S$ and $\{a, b\}$.

Definition. $S \subset c$ if and only if it is derivable from R1 through R7:

R1. $(S, v) \subset v$.

R2. If $(S, b) \subset c$ and $(S, b) \subset d$, then $(S, b) \subset c \wedge d$.

R3. If $(S, b, a) \subset c$, then $(S, b) \subset a \rightarrow c$.

R4. If $(R, d, e) \subset v$, then $(R, d \wedge e) \subset v$.

R5. If $(R, a \rightarrow d, a \rightarrow e) \subset v$, then $(R, a \rightarrow(d \wedge e)) \subset v$.

R6. If $(R,(a \wedge d) \rightarrow e) \subset v$, then $(R, a \rightarrow(d \rightarrow e)) \subset v$.

R7. If $(R, a \rightarrow v) \subset a$, then $(R, a \rightarrow v) \subset v$.

Lemma A. If $T \subset c$, then $(T, U) \subset c$.

Proof. In a given derivation of $T \subset c$, replace each instance $(S, v) \subset v$ of $\mathrm{R} 1$ by $(S, U, v) \subset v$. R2 through $\mathrm{R} 7$ carry $U$ along, so that the given derivation of $T \subset c$ becomes mutatis mutandis a derivation of $(T, U) \subset c$.

LEMMA B. If $T \subset b$ and $(S, b) \subset c$, then $(T, S) \subset c$.

Proof. The proof is by a double strong induction. The primary induction is on the rank of $b$. The secondary induction is on the number of applications of $\mathrm{R} 1$ through $\mathrm{R} 7$ in a given derivation of $(S, b) \subset c$. We first consider two special cases whose proofs do not depend on the induction framework.

Case $0 . b$ is in $S$. Then $(S, b) \subset c$ is $S \subset c$, and Lemma A gives $(T, S) \subset c$. From now on it is assumed that Case 0 does not hold.

Case 1. $(S, b) \subset c$ by $\mathrm{R} 1$. Then $c$ is a variable, and either $c$ is $b$ or $S$ is $\left(S_{1}, c\right)$. If $c$ is $b$, then Lemma A applied to $T \subset b$ gives $(T, S) \subset b$. If $S$ is $\left(S_{1}, c\right)$, then R1 gives $\left(T, S_{1}, c\right) \subset c$.

Basis. Suppose the rank of $b$ is zero, and the number of applications of $\mathrm{R} 1$ through $\mathrm{R} 7$ in the given derivation of $(S, b) \subset c$ is one. From the latter it follows that $(S, b) \subset c$ is inferred by $\mathrm{R} 1$, and Case 1 applies.

Induction step. Seven cases are considered, according as $(S, b) \subset c$ is inferred by $\mathrm{R} i, 1 \leqq i \leqq 7$. Case 1 takes care of $\mathrm{R} 1$.

Case 2. $(S, b) \subset c$ is inferred by R2. Then $c$ is $c_{1} \wedge c_{2}$, and $(S, b) \subset c_{1} \wedge c_{2}$ is inferred from $(S, b) \subset c_{1}$ and $(S, b) \subset c_{2}$. The SIH (secondary induction hypothesis) applied to $T \subset b$ and $(S, b) \subset c_{1}$ gives $(T, S) \subset c_{1}$. Similarly $(T, S) \subset c_{2}$. Then R2 gives $(T, S) \subset c_{1} \wedge c_{2}$.

Case 3. $(S, b) \subset c$ is inferred by R3. Then $c$ is $c_{1} \rightarrow c_{2}$, and $(S, b) \subset c_{1} \rightarrow c_{2}$ is inferred from $\left(S, b, c_{1}\right) \subset c_{2}$. The SIH applied to $T \subset b$ and $\left(S, b, c_{1}\right) \subset$ $c_{2}$ gives $\left(T, S, c_{1}\right) \subset c_{2}$, and then $\mathrm{R} 3$ gives $(T, S) \subset c_{1} \rightarrow c_{2}$. 
The conclusion of $\mathrm{R} i, 4 \leqq i \leqq 7$, is $(R, t) \subset v$, where $t$ is a term. Suppose $(S, b) \subset c$ is inferred by $R i, 4 \leqq i \leqq 7$. Then $c$ is a variable, and two cases arise, according as $b$ is or is not $t$. If $b$ is $t$ (and $S$ is $R$ ), then Case $i$ below applies. If $b$ is not $t$, then the proof follows the pattern of Cases 2 and 3: first apply the SIH, and then apply Ri. We illustrate with R4. Suppose $(S, b) \subset c$ is inferred by $\mathrm{R} 4$, and $b$ is not the term $d \wedge e$ that explicitly occurs in R4. Then $S$ is $\left(S_{1}, d \wedge e\right)$, and $\left(S_{1}, d \wedge e, b\right) \subset c$ is inferred from $\left(S_{1}, d, e, b\right) \subset c$. The SIH applied to $T \subset b$ and $\left(S_{1}, d, e, b\right) \subset c$ gives $\left(T, S_{1}, d, e\right) \subset c$, and then R4 gives $\left(T, S_{1}, d \wedge e\right) \subset c$.

Case 4. $(S, b) \subset c$ is inferred by $\mathrm{R} 4$ and $b$ is $d \wedge e$ and $S$ is $R$. Then $(S, d \wedge e) \subset c$ is inferred from $(S, d, e) \subset c . T \subset b$ is $T \subset d \wedge e$, and is necessarily inferred by $\mathrm{R} 2$ from $T \subset d$ and $T \subset e$. The PIH (primary induction hypothesis) applied to $T \subset d$ and $(S, d, e) \subset c$ gives $(T, S, e) \subset c$. Then the PIH applied to $T \subset e$ and $(T, S, e) \subset c$ gives $(T, S) \subset c$.

Case 5. $(S, b) \subset c$ is inferred by R5, and $b$ is $a \rightarrow(d \wedge e)$ and $S$ is $R$. Then $(S, a \rightarrow(d \wedge e)) \subset c$ is inferred from $(S, a \rightarrow d, a \rightarrow e) \subset c . T \subset b$ is $T \subset a \rightarrow$ $(d \wedge e)$, and is necessarily inferred from $(T, a) \subset d \wedge e$ by $\mathrm{R} 3$. The latter is necessarily inferred by $\mathrm{R} 2$ from $(T, a) \subset d$ and $(T, a) \subset e$. R3 applied to $(T, a) \subset d$ gives $T \subset a \rightarrow d$. Similarly $T \subset a \rightarrow e$. The PIH applied to $T \subset$ $a \rightarrow d$ and $(S, a \rightarrow d, a \rightarrow e) \subset c$ gives $(T, S, a \rightarrow e) \subset c$. Then the PIH applied to $T \subset a \rightarrow e$ and $(T, S, a \rightarrow e) \subset c$ gives $(T, S) \subset c$.

Case 6. $(S, b) \subset c$ is inferred by R6, and $b$ is $a \rightarrow(d \rightarrow e)$ and $S$ is $R$. Then $(S, a \rightarrow(d \rightarrow e)) \subset c$ is inferred from $(S,(a \wedge d) \rightarrow e) \subset c . T \subset b$ is $T \subset a \rightarrow$ $(d \rightarrow e)$, and is necessarily inferred by $\mathrm{R} 3$ from $(T, a) \subset d \rightarrow e$. The latter is necessarily inferred by $\mathrm{R} 3$ from $(T, a, d) \subset e$. R4 gives $(T, a \wedge d) \subset e$, and then R3 gives $T \subset(a \wedge d) \rightarrow e$. The SIH applied to $T \subset(a \wedge d) \rightarrow e$ and $(S,(a \wedge d) \rightarrow e) \subset c$ gives $(T, S) \subset c$.

Case 7. $(S, b) \subset c$ is inferred by R7, and $b$ is $a \rightarrow c$ and $S$ is $R$. Then $(S, a \rightarrow c) \subset c$ is inferred from $(S, a \rightarrow c) \subset a . T \subset b$ is $T \subset a \rightarrow c$. The SIH applied to $T \subset a \rightarrow c$ and $(S, a \rightarrow c) \subset a$ gives $(T, S) \subset a . T \subset a \rightarrow c$ is necessarily inferred by R3 from $(T, a) \subset c$. The PIH applied to $(T, S) \subset a$ and $(T, a) \subset c$ gives $(T, S) \subset c$.

Lemma C. If $(S, d, e) \subset c$, then $(S, d \wedge e) \subset c$.

Lemma D. If $(S, a \rightarrow d, a \rightarrow e) \subset c$, then $(S, a \rightarrow(d \wedge e)) \subset c$.

Lemma E. If $(S,(a \wedge d) \rightarrow e) \subset c$, then $(S, a \rightarrow(d \rightarrow e)) \subset c$.

Proofs. Lemma $\mathrm{D}$ is proved by strong induction on the rank $n$ of $c$. If $n=0$, then $c$ is a variable $v$, and R5 gives the desired result. For the induction step, we consider two cases, according as $c$ is $c_{1} \wedge c_{2}$ or $c$ is $c_{1} \rightarrow c_{2}$. Suppose $c$ is $c_{1} \wedge c_{2}$, and $(S, a \rightarrow d, a \rightarrow e) \subset c_{1} \wedge c_{2}$. The latter is 
necessarily inferred by $\mathrm{R} 2$ from $(S, a \rightarrow d, a \rightarrow e) \subset c_{1}$ and $(S, a \rightarrow d, a \rightarrow e) \subset$ $c_{2}$. The induction hypothesis gives $(S, a \rightarrow(d \wedge e)) \subset c_{1}$ and $(S, a \rightarrow(d \wedge e)) \subset$ $c_{2}$, and then R2 gives $(S, a \rightarrow(d \wedge e)) \subset c_{1} \wedge c_{2}$. Suppose $c$ is $c_{1} \rightarrow c_{2}$, and $(S, a \rightarrow d, a \rightarrow e) \subset c_{1} \rightarrow c_{2}$. The latter is necessarily inferred by $\mathrm{R} 3$ from $\left(S, a \rightarrow d, a \rightarrow e, c_{1}\right) \subset c_{2}$. The induction hypothesis gives $\left(S, a \rightarrow(d \wedge e), c_{1}\right) \subset$ $c_{2}$, and then R3 gives $(S, a \rightarrow(d \wedge e)) \subset c_{1} \rightarrow c_{2}$. Lemmas $\mathrm{C}$ and $\mathrm{E}$ are proved in exactly the same way, with the basis steps given by $\mathrm{R} 4$ and $\mathrm{R} 6$ respectively.

LEMMA F. $(S, c) \subset c$. If $T \subset a$, then $(T, a \rightarrow c) \subset c$.

Proof. The two parts are proved simultaneously by strong induction on the rank $n$ of $c$. If $n=0$, then $c$ is a variable $v$. R1 gives $(S, v) \subset v$. Suppose $T \subset a$. Then Lemma A gives $(T, a \rightarrow v) \subset a$, and $\mathrm{R} 7$ gives $(T, a \rightarrow v) \subset v$. For the induction step we consider two cases, according as $c$ is $c_{1} \wedge c_{2}$ or $c$ is $c_{1} \rightarrow c_{2}$. Suppose $c$ is $c_{1} \wedge c_{2}$. The induction hypothesis gives $\left(S, c_{1}, c_{2}\right) \subset c_{1}$ and $\left(S, c_{1}, c_{2}\right) \subset c_{2}$, and then $\mathrm{R} 2$ gives $\left(S, c_{1}, c_{2}\right) \subset$ $c_{1} \wedge c_{2}$, and then Lemma $C$ gives $\left(S, c_{1} \wedge c_{2}\right) \subset c_{1} \wedge c_{2}$. Suppose $T \subset a$. The induction hypothesis gives $\left(T, a \rightarrow c_{1}\right) \subset c_{1}$. Lemma A gives $\left(T, a \rightarrow c_{1}\right.$, $\left.a \rightarrow c_{2}\right) \subset c_{1}$. Similarly $\left(T, a \rightarrow c_{1}, a \rightarrow c_{2}\right) \subset c_{2}$. R2 gives $\left(T, a \rightarrow c_{1}, a \rightarrow c_{2}\right) \subset$ $c_{1} \wedge c_{2}$, and then Lemma $\mathrm{D}$ gives $\left(T, a \rightarrow\left(c_{1} \wedge c_{2}\right)\right) \subset c_{1} \wedge c_{2}$. Suppose $c$ is $c_{1} \rightarrow c_{2}$. The induction hypothesis gives $\left(S, c_{1}\right) \subset c_{1}$. Then the induction hypothesis gives $\left(S, c_{1}, c_{1} \rightarrow c_{2}\right) \subset c_{2}$, and then R3 gives $\left(S, c_{1} \rightarrow c_{2}\right) \subset$ $c_{1} \rightarrow c_{2}$. Suppose $T \subset a$. Lemma A gives $\left(T, c_{1}\right) \subset a$. The induction hypothesis gives $\left(T, c_{1}\right) \subset c_{1}$. Then R2 gives $\left(T, c_{1}\right) \subset a \wedge c_{1}$. Then the induction hypothesis gives $\left(T, c_{1},\left(a \wedge c_{1}\right) \rightarrow c_{2}\right) \subset c_{2}$. R3 gives $\left(T,\left(a \wedge c_{1}\right) \rightarrow c_{2}\right) \subset c_{1} \rightarrow c_{2}$, and then Lemma E gives $\left(T, a \rightarrow\left(c_{1} \rightarrow c_{2}\right)\right) \subset c_{1} \rightarrow c_{2}$.

LEMMA G. If $a \leqq b$, then $a \subset b .(a \subset b$ means $\{a\} \subset b$. $)$

Proof. It suffices to show that (1) through (6) continue to hold if $\leqq$ is replaced throughout by $\subset$. For (1), Lemma $\mathrm{F}$ gives $a \subset a$. Lemma $\mathrm{B}$, with $T=\{a\}$ and $S$ empty, gives (2). For (3), Lemma F gives $\{a, b\} \subset a$, and then Lemma $C$ gives $a \wedge b \subset a$. Similarly $a \wedge b \subset b$. R2 gives (4), and $\mathrm{R} 3$ gives (5). For (6), Lemma $\mathrm{F}$ gives $a \subset a$. Lemma $\mathrm{F}$ gives $\{a, a \rightarrow b\} \subset b$, and then Lemma $C$ gives $a \wedge(a \rightarrow b) \subset b$.

LemMA H. If $\left\{a_{1}, \cdots, a_{k}\right\} \subset b$, then $a_{1} \wedge \cdots \wedge a_{k} \leqq b$. In particular, if $a \subset b$, then $a \leqq b$.

Proof. It suffices to show that R1 through R7 continue to hold if $\left\{a_{1}, \cdots, a_{k}\right\} \subset b$ is replaced throughout by $a_{1} \wedge \cdots \wedge a_{k} \leqq b$. (3) gives $\mathrm{R} 1$ if $S$ is not empty, and (1) gives R1 if $S$ is empty. (4) gives R2. (5) gives R3. R4 is obvious. (8) gives R5; (9) gives R6. R7 follows from (10). 
THEOREM. There is an effective procedure for deciding, for any terms $a$ and $b$ in the theory of implicative semilattices, whether or not the equation $a=b$ is an identity.

Proof. By a previous remark, the problem reduces to whether or not $a \leqq b$ and $b \leqq a$. By Lemmas $\mathrm{G}$ and $\mathrm{H}, a \leqq b$ if and only if $a \subset b$. Let R $k^{*}$, $2 \leqq k \leqq 7$, be the inverse of $\mathrm{R} k$. (Interchange the if and then parts.) The procedure is to start with $a \subset b$ and work backwards via the inverse rules in an attempt to find a derivation of $a \subset b$. Consider a derivation to be in tree form. The tops are all instances of R1; the bottom is $a \subset b$; R2 gives rise to branching. Call $S \subset c$ an inequality. A branch is a finite sequence $I_{1}, \cdots, I_{n}$ of inequalities such that for each $j, 1 \leqq j<n, I_{j+1}$ is inferred from $I_{j}$ by one of R2 through R7. (In the case of R2, $I_{j+1}$ is inferred from $I_{j}$ and one other inequality.) A derivation is irredundant if and only if no inequality occurs more than once in the same branch. (The inequalities $S \subset c$ and $T \subset d$ are the same if and only if $c$ is $d$ and $S$ is the same set as $T$.) Clearly it suffices to consider only irredundant derivations.

Starting with $a \subset b$, a mixture of $\mathrm{R}^{*}$ and $\mathrm{R} 3^{*}$ gives rise to one or more branches topped by inequalities of the form $S \subset v$, where $v$ is a variable. Consider one such branch. A mixture of R4* through R6* reduces each term on the left to one or more terms of the form $u$ or $a \rightarrow u$, where $u$ is a variable. If one of these terms is $v$, then $\mathrm{R} 1$ applies and the branch terminates. If none of these terms is $v$, but one of them is of the form $a \rightarrow v$, then R7* applies, the term $a$ moves to the right, and a new cycle begins. If neither $\mathrm{R} 1$ nor $\mathrm{R}^{*}$ applies, then the branch aborts. It may happen

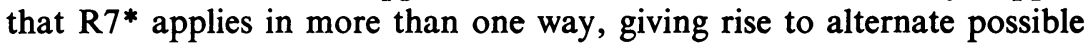
derivations. Each possible derivation must be pursued until one derivation terminates or all possible derivations abort.

It remains to show that the procedure terminates, i.e. each possible derivation is finite, and there are only finitely many possible derivations. Since only irredundant derivations need be considered, it suffices to show that only finitely many inequalities can be generated by starting with $a \subset b$ and applying the inverse rules. To this end, let $v_{1}, \cdots, v_{k}$ be all the distinct variables that occur in $a$ or $b$, and let $n$ be the greater of the ranks of $a$ and $b$. Let $A$ be the set of all terms of rank not exceeding $n$ which contain no variable not in the list $v_{1}, \cdots, v_{k}$. Clearly $A$ is a finite set, say with $m$ elements. Call an inequality $S \subset c$ an $A$-inequality if $c$ and every term in $S$ are in $A$. Inspection shows that $a \subset b$ is an $A$-inequality, and each inverse rule applied to an $A$-inequality yields one or two $A$-inequalities. Hence only $A$-inequalities can occur in any possible derivation of $a \subset b$. In an $A$-inequality $S \subset c$, there are $m$ choices for $c$ since $c$ is in $A$, and there 
are $2^{m}$ choices for $S$ since $S$ is a finite subset of $A$. Hence there are only (m) $\left(2^{m}\right) A$-inequalities, and we are done.

The decision procedure above is based on ideas of Gentzen [2] as set forth in Kleene [3]. Modifications have been made to increase speed and efficiency by minimizing branching and alternate derivations. The chief novelty is that Gentzen's two-premise left $\rightarrow$ rule is replaced by the onepremise rule $\mathbf{R} 7$.

\section{REFERENCES}

1. H. B. Curry, Foundations of mathematical logic, McGraw-Hill, New York, 1963. MR 26 \#6036.

2. Gerhard Gentzen, Untersuchungen über das logische Schliessen, Math. Z. 39 (1934/35), 176-210, 405-431; English transl., in M. E. Szabo, The collected papers of Gerhard Gentzen, Studies in Logic and the Foundations of Math., North-Holland, Amsterdam, 1969, pp. 68-103. MR 41 \#6660.

3. S. C. Kleene, Introduction to metamathematics, Van Nostrand, New York, 1952. MR 14, 525.

Department of Mathematics, Southwestern at Memphis, Memphis, Tennessee 38112 